\title{
Cone-Size Dependence of Jet Suppression in Heavy-Ion Collisions
}

\author{
Yacine Mehtar-Tani* \\ RIKEN BNL Research Center and Physics Department, Brookhaven National Laboratory, Upton, New York 11973, USA \\ Daniel Pablos ${ }^{\dagger}$ and Konrad Tywoniuk ${ }^{\ddagger}$ \\ Department of Physics and Technology, University of Bergen, 5007 Bergen, Norway
}

(Received 8 February 2021; accepted 23 November 2021; published 17 December 2021)

\begin{abstract}
The strong suppression of high- $p_{T}$ jets in heavy-ion collisions is a result of elastic and inelastic energy loss suffered by the jet multiprong collection of color charges that are resolved by medium interactions. Hence, quenching effects depend on the fluctuations of the jet substructure that are probed by the cone-size dependence of the spectrum. In this Letter, we present the first complete, analytic calculation of the inclusive $R$-dependent jet spectrum in $\mathrm{PbPb}$ collisions at LHC energies, including resummation of energy loss effects from hard, vacuumlike emissions occurring in the medium and modeling of soft energy flow and recovery at the jet cone. Both the geometry of the collision and the local medium properties, such as the temperature and fluid velocity, are given by a hydrodynamic evolution of the medium, leaving only the coupling constant in the medium as a free parameter. The calculation yields a good description of the centrality and $p_{T}$ dependence of jet suppression for $R=0.4$ together with a mild cone-size dependence, which is in agreement with recent experimental results. Gauging the theoretical uncertainties, we find that the largest sensitivity resides in the leading logarithmic approximation of the phase space of resolved splittings, which can be improved systematically, while nonperturbative modeling of the soft-gluon sector is of relatively minor importance up to large cone sizes.
\end{abstract}

DOI: 10.1103/PhysRevLett.127.252301

Introduction.-Jets are collimated sprays of energetic particles produced in collider experiments that act as proxies of accelerated quark and gluon degrees of freedom originating from elementary large momentum-transfer processes or decays of massive bosons. In this context, precision computations of QCD jet events play a crucial role in a wide range of fundamental measurements at colliders [1,2], including measurements of the Higgs boson properties [3] and searches beyond the standard model.

In contrast, jet physics in heavy-ion collisions probes the discovery frontier to potentially reveal and detail new emergent QCD phenomena in dense partonic systems. The creation of a short-lived, hot, and dense state of deconfined matter, also known as the quark-gluon plasma (QGP), leaves a strong imprint on high- $p_{T}$ probes [4,5]. This phenomenon, commonly referred to as "jet quenching," was observed for the first time at the Relativistic Heavy Ion Collider (RHIC) [6-8] and later at the LHC [9-15]. Currently, the exact mechanisms responsible for jet modifications, including details of the energy transport

Published by the American Physical Society under the terms of the Creative Commons Attribution 4.0 International license. Further distribution of this work must maintain attribution to the author(s) and the published article's title, journal citation, and DOI. Funded by SCOAP ${ }^{3}$. from high-energy to low-energy modes and color decoherence of multipartonic states, are under intense investigation.

The basic mechanisms of parton energy loss were understood and formalized in the 1990s [16-20]. A number of approximations were then necessary to allow for analytic computations. This introduced a theoretical bias on model calculations, absent from full numerical approaches [21-23], that could be alleviated by incorporating the two main scattering regimes: the Rutherford scattering regime, dominated by a single hard momentum transfer (included in earlier analytical attempts [24,25]), and the low momentum regime where multiple scatterings contribute with order one probability. Furthermore, with the measurements of fully reconstructed jets at the LHC and RHIC, it was soon recognized that higher order corrections accounted for by parton cascades are not negligible. In parallel to the development of Monte Carlo (MC) event generators [26-36] tremendous conceptual progress has been made in addressing these questions by analyzing the interference structure of two successive splittings within the medium [37-41]. To leading logarithmic accuracy, the in-medium jet evolution is characterized by an early vacuum parton cascade whose constituents are resolved by the medium due to color decoherence, whereas unresolved splittings factorize from the in-medium evolution, losing energy coherently as a single color charge $[42,43]$. This work aims to 
address these two challenges within a first-principle analytic framework.

In this Letter, we report a first-principle calculation of the single-inclusive jet spectrum and its cone-size dependence in heavy-ion collisions where high density effects are resummed to all orders. Even though jets with a larger cone do retain a larger fraction of the lost energy, a priori reducing jet suppression compared to a smaller one, we show that resumming the additional energy loss induced by the cone-size dependent jet substructure fluctuations yields a final jet suppression that is very mildly dependent on $R$. The well established connection between energy loss dynamics and coherence effects, which determine the actual resolved phase space of the jet in the medium, allows us to confront our results with high-statistics experimental data merely by constraining the strength of the QCD coupling in the medium. Additional fluctuations on the path and medium density explored by the jet, which vary event by event, are taken care of by embedding our framework into a realistic heavy-ion environment in which the medium is described by the explosion of a liquid droplet of deconfined QCD matter. Finally, we aim to assess what the relative magnitude of the uncertainties associated with describing the perturbative structure and the soft, infrared features of medium-modified jets are as a function of their kinematics. Providing a quantitative answer to this question is crucial if one aims to establish the predictive power of weak coupling techniques in jet quenching phenomenology.

Theoretical formalism. - The spectrum of jets with conesize $R$ in proton-proton collisions is given by the convolution of the initial hard parton spectra with the corresponding fragmentation function. The latter describes the energy remaining within the jet at different angular resolutions $R$, starting from a large value $R_{0} \sim 1$. For a steeply falling initial spectrum, it can be written as [44] $\sigma^{p p}\left(p_{T}, R\right)=$ $\sum_{k=q, g} f_{\text {jet } / k}^{(n-1)}\left(R \mid p_{T}, R_{0}\right) \hat{\sigma}_{k}\left(p_{T}, R_{0}\right)$, where $n \equiv n_{k}\left(p_{T}, R_{0}\right)$ is the power index of the cross-section of the hard parton with flavor $k$ and $\hat{\sigma}_{k}\left(p_{T}, R_{0}\right)$ is evaluated at leading order. The moment of the fragmentation function of an initial hard parton with flavor $k$, i.e., $f_{\text {jet } / k}^{(n)}\left(R \mid p_{T}, R_{0}\right)=$ $\int_{0}^{1} d x x^{n} f_{\text {jet } / k}\left(R \mid x, R_{0}\right)$, gets both quark and gluon contributions, $f_{\text {jet } / k}^{(n)}=\sum_{i=q, g} f_{i / k}^{(n)}$, due to flavor conversion during the Dokshitzer-Gribov-Lipatov-Altarelli-Parisi (DGLAP) evolution [44-47].

Correspondingly, the cross section in nucleusnucleus collisions $(A A)$ is convolved with a probability distribution $P(\epsilon)$ describing medium-induced energy loss out of the jet cone, and reads $\sigma^{A A}\left(p_{T}, R\right)=$ $\sum_{i=q, g} \int_{0}^{\infty} d \epsilon P_{i}(\epsilon) \tilde{\sigma}_{i}^{p p}\left(p_{T}+\epsilon, R\right)$, where $\tilde{\sigma}_{i}^{p p}$ corresponds to the quark-gluon contribution to the total cross section [the tilde serves as a reminder that the proton parton distribution functions (PDFs) are replaced by nuclear PDFs]. Finally, the flavor dependent resummed quenching factors $\quad Q_{i}\left(p_{T}, R\right) \equiv \int_{0}^{\infty} d \epsilon P_{i}(\epsilon) \tilde{\sigma}_{i}^{p p}\left(p_{T}+\epsilon\right) / \tilde{\sigma}_{i}^{p p}\left(p_{T}\right) \simeq$ $\left.\int_{0}^{\infty} d \epsilon P(\epsilon) e^{-\nu \epsilon}\right|_{\nu=n / p_{T}}$, where in the last step we used the approximation of large power index $n$, account for the energy loss by a jet with momentum $p_{T}$ and size $R$ during the passage of a background medium [48]. This factorization is justified by the fact that out of cone vacuum evolution takes place at much shorter times than energy loss and was used as a basis for the extraction of the quenching weights from the data $[49,50]$.

A novel ingredient of our setup are the quenching factors $Q_{i}\left(p_{T}, R\right)$ that resum contributions to the total energy loss of a jet consisting of many color charges that interact with the medium. The magnitude of this effect can be gauged by comparing the formation time of a splitting, $t_{f}=$ $2 /\left[z(1-z) p_{T} \theta^{2}\right]$, to the characteristic timescale the medium needs to resolve the product of the splitting, namely $t_{d}=\left[\hat{q}_{0} \theta^{2} / 12\right]^{-1 / 3}[37-40]$. Here, $\hat{q}_{0} \equiv d\left\langle k_{\perp}^{2}\right\rangle / d t$ is the transport coefficient that encodes medium properties, the so-called jet quenching parameter [17]. Hence, jet splittings occurring at timescales much shorter than the related medium timescale, that is if $t_{f} \ll t_{d} \ll L$, obey the same properties as vacuum splittings $[42,43]$. The latter inequality implies that a splitting with $\theta<\theta_{c}$, where the critical angle is $\theta_{c}=\left(\hat{q}_{0} L^{3} / 12\right)^{-1 / 2}$, will not be resolved by the medium. With these considerations in mind, one can show that in the large $n$ limit, since the convolution of energy loss probability distributions reduces to a direct product of quenching factors (in Laplace space), the evolution equation for the resummed quenching weight is [42]

$$
\begin{aligned}
\frac{\partial Q_{i}(p, \theta)}{\partial \ln \theta}= & \int_{0}^{1} d z \frac{\alpha_{s}\left(k_{\perp}\right)}{2 \pi} p_{j i}^{(k)}(z) \Theta_{\mathrm{res}}(z, \theta) \\
& \left.\times\left[Q_{j}(z p, \theta) Q_{k}((1-z) p, \theta)\right)-Q_{i}(p, \theta)\right],
\end{aligned}
$$

where $k_{\perp}=z(1-z) p \theta, \quad p_{j i}^{(k)}(z)$ are the unregularized Altarelli-Parisi splitting functions and the phase space constraint is given by $\Theta_{\text {res }}(p, R)=\Theta\left(t_{f}<t_{d}<L\right)$. Above, it is understood that $p$ is evaluated at $p \equiv p_{T}$. This distinction is necessary when solving Eq. (1) since the initial condition also depends on $p_{T}$. The nonlinear evolution equations account for the energy loss of the multiprong jet substructures that are generated by early collinear splittings.

The initial conditions for the resummed quenching factors $Q_{i}(p, R)$ at $R=0$ are the bare quenching factors for single partons. In this work, we have $Q_{i}(p, 0)=$ $Q_{\mathrm{rad}, i}^{(0)}\left(p_{T}\right) Q_{\mathrm{el}, i}^{(0)}\left(p_{T}\right)$, where the two bare quenching factors are the LT of the corresponding probability distributions that describe radiative and elastic energy loss [48,51], For their precise definitions, see below. The radiative and elastic energy loss are driven by the transport coefficients $\hat{q}$ and $\hat{e}$ [52], respectively, which are related by the fluctuation-dissipation relation $\hat{e}=\hat{q} /(4 T)$ in a weakly 
coupled plasma (where $\hat{e}_{g}=\hat{e}$ for gluons, and $\hat{e}_{q}=$ $\left(C_{F} / N_{c}\right) \hat{e}_{g}$ for quarks) [53]. The quenching factor due to radiative energy loss off a single parton is simply the exponential of the LT for a single-inclusive gluon radiative spectrum [48,51], which takes into account the broadening of emitted partons and rapid thermalization of the lost energy [54-58].

The medium-induced gluon radiation spectrum has been computed up to next-to-leading order (NLO) within the improved opacity expansion (IOE) in the soft limit [59-61] and unifies both the Baier-Dokshitzer-Mueller-PeignéSchiff approach with the Gyulassy-Levai-Vitev (GLV)higher-twist formalism $[62,63]$, which has proven to be an important ingredient for phenomenological studies [64,65]. It is expressed as $d I_{\mathrm{NLO}} / d \omega=d I^{(0)} / d \omega+d I^{(1)} / d \omega$, with

$$
\begin{gathered}
\frac{d I^{(0)}}{d \omega}=\frac{2 \alpha_{s} C_{R}}{\pi \omega} \ln |\cos \Omega L|, \\
\frac{d I^{(1)}}{d \omega}=\frac{\alpha_{s} C_{R} \hat{q}_{0}}{2 \pi} \operatorname{Re} \int_{0}^{L} d s \frac{-1}{k^{2}(s)} \ln \frac{-k^{2}(s)}{Q^{2} e^{-\gamma_{E}}},
\end{gathered}
$$

where $\quad \Omega=(1-i) \sqrt{\hat{q} /(4 \omega)}, \quad k^{2}(s)=i \omega \Omega[\cot \Omega s-$ $\tan \Omega(L-s)] / 2$, and the strong coupling constant runs with the typical transverse momentum of the emission, i.e., $\alpha_{s}=\alpha_{s}\left((\hat{q} \omega)^{1 / 4}\right)$ [66]. In this expansion, the effective transport coefficient $\hat{q}$ differs from the bare $\hat{q}_{0}$ by a factor that reflects the full leading logarithmic contribution, i.e., $\hat{q}=\hat{q}_{0} \ln \left(Q^{2} / \mu_{*}^{2}\right)$, where $\hat{q}_{0}=g_{\text {med }}^{2} N_{c} m_{D}^{2} T /(4 \pi)$ for a thermal medium in the hard thermal loop (HTL) theory and the lower cutoff scale is $\mu_{*}^{2}=m_{D}^{2} \exp \left[-2+2 \gamma_{E}\right] / 4$ $[60,61]$. The effective medium scale $Q^{2}$ depends itself on the amount of rescattering in the medium and can be found by solving the transcendental equation $Q^{4}=\hat{q}_{0} \omega \ln Q^{2} / \mu_{*}^{2}$ [67] (a similar idea was also presented in [68]). In our framework, the medium coupling $g_{\text {med }}$, the only free parameter that determines energy loss, is to be extracted from the comparison to experimental data. Note that we work in the eikonal approximation and therefore neglect suppressed powers of the inverse jet energy, which are sensitive to the flow of the medium and its spatial gradients as pointed out recently in [69-71].

We first consider semihard gluons that are emitted within the range $\omega_{s}<\omega \lesssim \omega_{c}$, where $\omega_{c} \equiv \hat{q}_{0} \ln \left(\hat{q}_{0} L / \mu_{*}^{2}\right) L^{2} / 2$ corresponds to the maximal energy accumulated through multiple soft scatterings, and $\omega_{s} \equiv\left[g_{\text {med }}^{2} N_{c} /(2 \pi)^{2}\right]^{2} \pi \hat{q}_{0} L^{2}$ is the energy scale at which the emission probability is of order one, determining the onset of turbulent energy loss [72]. Their broadening distribution reflects the typical transverse momentum kicks received in the plasma. Softer gluons, with $\omega \ll \omega_{c}$ and small transverse kicks $k_{\perp}^{2} \sim \hat{q} L$, will experience Gaussian broadening, while harder emissions, with $\omega \gg \omega_{c}$ and typically large transverse momenta $k_{\perp}^{2}>\omega / L \gg \hat{q} L$, are governed by a power-law behavior, $\sim \hat{q}_{0} L / k_{\perp}^{4}$. We assume that the effect of broadening appears as a multiplicative factor $B\left(\omega R ; Q_{\text {broad }}^{2}\right)=(d I / d \omega)^{-1} \int_{(\omega R)^{2}}^{\infty} d k_{\perp}^{2} d I /\left(d \omega d k_{\perp}^{2}\right)$, representing the probability for the emitted gluon to be transported out to an angle larger than the jet cone [73], $\theta>R$, where $Q_{\text {broad }}^{2}$ denotes a characteristic broadening scale. The full out-of-cone spectrum consists of two terms, from the IOE expansion up to NLO with their corresponding broadening factors [55], such that

$$
\begin{aligned}
\frac{d I_{>}}{d \omega}= & B\left(\omega R ; Q_{s}^{2} / 2\right) \frac{d I^{(0)}}{d \omega} \\
& +B\left(\omega R ; \max \left[Q_{s}^{2}, 16 \omega /\left(\pi^{2} L\right)\right]\right) \frac{d I^{(1)}}{d \omega} .
\end{aligned}
$$

Since emissions can take place anywhere along the inmedium path, one also has to average over the radiation time. This is approximated by simply setting $Q_{\text {broad }}^{2}=$ $\hat{q} L / 2[54,55]$ in the first term. The choice of scale in the second term reproduces the correct behavior of the full GLV spectrum in the high $\omega$ and $k_{\perp}$ regime up to a logarithmic factor, see Supplemental Material [74] for more details.

Soft gluons, with $T<\omega<\omega_{s}$, cascade quasi-instantaneously to the thermal scale [72] and should effectively be treated within hydrodynamics. Their emission rate is therefore not affected by transverse momentum broadening. Assuming that their distribution becomes approximately uniform in the solid angle around the jet, we account for the possibility that a fraction of this energy ends up back in the jet cone by modifying $\omega \rightarrow \omega\left(1-\left(R / R_{\text {rec }}\right)^{2}\right)$, where the recovery angle $R_{\text {rec }}$ is a free parameter [83]. An analogous modification is applied to the elastic quenching factor. Emissions at $\omega<T$ belong to the Bethe-Heitler regime, and are not relevant for our present phenomenological application $[65,88]$.

Putting all the pieces together, the final expression for the radiative bare quenching factor reads

$$
\begin{aligned}
Q_{\mathrm{rad}}^{(0)}\left(p_{T}\right)= & \exp \left[-\int_{\omega_{s}}^{\infty} d \omega \frac{d I_{>}}{d \omega}\left(1-e^{-\nu \omega}\right)\right. \\
& \left.-\int_{T}^{\omega_{s}} d \omega \frac{d I^{(0)}}{d \omega}\left(1-e^{-\nu \omega\left(1-\left(\frac{R}{\mathrm{R}_{\mathrm{rec}}}\right)^{2}\right)}\right)\right],
\end{aligned}
$$

where the parton flavor index is implicit and $\nu \equiv n / p_{T}$. We have approximated $d I_{\mathrm{NLO}} / d \omega \simeq d I^{(0)} / d \omega$ in the soft regime. The bare quenching factor for elastic energy loss is $Q_{\mathrm{el}}^{(0)}\left(p_{T}\right)=\exp \left[-\hat{e} L \nu\left(1-\left(R / R_{\mathrm{rec}}\right)^{2}\right)\right]$, also with implicit parton flavor dependence. It results from taking the LT of $\delta\left(\epsilon-\hat{e} L\left(1-R^{2} / R_{\mathrm{rec}}^{2}\right)\right)$. We shall see that our results at small cone sizes are not very sensitive to the above modeling of energy recovery. 
TABLE I. Summary of the effect of relative change of $R_{A A}$ from varying key parameters for cone sizes $R=0.2-0.6$; see text for further details.

\begin{tabular}{lcc}
\hline \hline Parameter & Variation & Effect \\
\hline$\theta_{c}$ & {$\left[\theta_{c} / 2,2 \theta_{c}\right]$} & $\lesssim 20 \%$ \\
IOE & LO $/ \mathrm{NLO}$ & $\sim 2 \%$ \\
$n$ & \pm 1 & $\sim 10 \%$ \\
$R_{\text {rec }}$ & {$[1, \infty]$} & $\lesssim 10 \%$ \\
$\omega_{s}$ & {$\left[\omega_{s} / 2,2 \omega_{s}\right]$} & $\lesssim 8 \%$ \\
\hline \hline
\end{tabular}

Numerical results.-Using the bare quenching factors for the radiative and elastic contributions to energy loss, we numerically solve the coupled evolution equations in Eq. (1). The cone-size dependence of the bare quenching factors, resulting in more energy loss for smaller $R$, are to a large extent washed away by the evolution.

In our numerical computations, we fix the values of the two free parameters of our setup, $g_{\text {med }}$ and $R_{\text {rec }}$. The energy recovery parameter $R_{\text {rec }}$ has been varied between $R_{\text {rec }}=$ $\pi / 2$ and $R_{\mathrm{rec}}=(5 / 6) \pi / 2$, which was estimated from a linearized approach to model the QGP wake for $[84,89]$. To constrain $g_{\text {med }}$ we have compared our results for the widely used nuclear suppression factor for jet production, also known as $R_{A A}$, for jets with $R=0.4$ around $p_{T} \approx 100 \mathrm{GeV}$ against high-statistics experimental data from ATLAS for the $0 \%-10 \%$ centrality class of $\mathrm{PbPb}$ collisions at $\sqrt{s}=$ $5.02 \mathrm{ATeV}$ [90]. In order to compute $R_{A A}$, we have taken the ratio between the nuclear and the vacuum spectra, which comprise a weighted sum of the quark and gluon jet contributions to the full spectrum [91]. Event-by-event inmedium path fluctuations of a jet through the QGP have been taken into account by embedding our framework into a realistic heavy-ion environment as simulated in the VISHNU hydrodynamical model [95], see Supplemental Material [74] for further details. The value of $g_{\text {med }}$ is thus constrained by the experimental data to be within the range $g_{\text {med }} \in\{2.2,2.3\}$. We emphasize that the magnitude of quenching is predominantly driven by the emission of copious soft gluons [48], see also Table I. The extracted parameters yield an average value $\left\langle\hat{q}_{0}\right\rangle \simeq 0.45 \mathrm{GeV}^{2} / \mathrm{fm}$ in $0 \%-10 \%$ central $\mathrm{PbPb}$ collisions that is well within the perturbative regime; see Supplemental Material [74] for more information on the centrality dependence of key parameters. The obtained value for the bare quenching parameter is consistent with previous [96] and more recent studies [97] of parton energy loss phenomenology. However, the logarithmic corrections to the bare medium parameters, resulting in $Q^{2}=14.2 \mathrm{GeV}^{2}$ for the factorization scale and $\hat{q}=2.46 \mathrm{GeV}^{2} / \mathrm{fm}$, produce a relatively large maximal medium energy scale $\omega_{c} \approx 73 \mathrm{GeV}$. The energy scale governing the onset of the turbulent regime is in central collisions $\omega_{s} \approx 19 \mathrm{GeV}$.

We show results for $R_{A A}$ as a function of jet $p_{T}$ and centrality in Fig. 1, confronted against data from

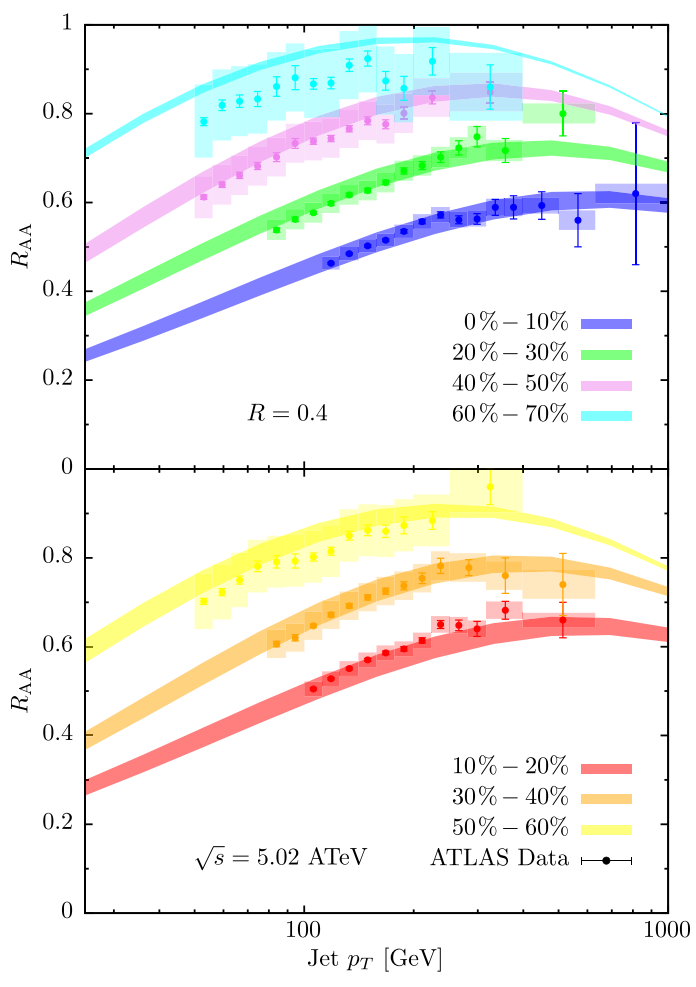

FIG. 1. Calculation of inclusive jet $R_{A A}$ in $\mathrm{PbPb}$ collisions at $\sqrt{s}=5.02 \mathrm{ATeV}$, compared to ATLAS data [90], for different centralities.

ATLAS [90] for $R=0.4$ jets. We can see that our theoretical results, constrained to describe $R_{A A}$ around $p_{T} \approx$ $100 \mathrm{GeV}$ for $0 \%-10 \%$ only, give an excellent description of both the $p_{T}$ dependence and centrality evolution of jet suppression. We note that the downturn of $R_{A A}$ at the highest jet $p_{T}$ is due to the nuclear PDF modifications imprinted in the initial hard parton spectra of $\mathrm{PbPb}$ collisions, as already noted in $[86,98,99]$. Finally, we quantify the $R$ (in)dependence of jet suppression by taking double ratios of the full results for $R_{A A}$ as in Fig. 2, with the largest size $R=1$ in the denominator. Such notably mild

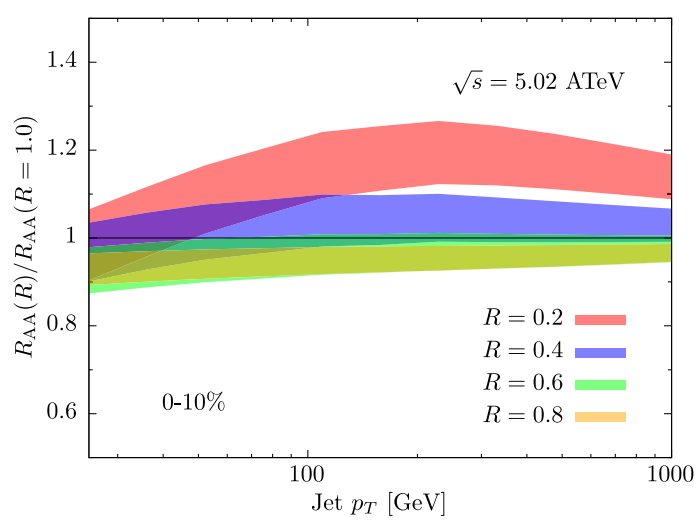

FIG. 2. Double ratio of inclusive jet $R_{A A}$ for different jet radius $R$ over $R_{A A}$ for $R=1.0$ in $\mathrm{PbPb}$ collisions at $\sqrt{s}=5.02 \mathrm{ATeV}$. 
dependence of jet suppression with $R$ is in agreement with ALICE results at low- $p_{T}[100]$ and with recent experimental preliminary data from CMS at high- $p_{T}$ [101].

Summary and discussion.-We have provided a first, analytical description of the cone-size dependent jet spectrum in heavy-ion collisions at the LHC implemented in a realistic event-by-event setup including nuclear geometry and hydrodynamic expansion of the quark-gluon plasma, and demonstrated how the cone-size dependent jet spectrum is largely governed by the energy loss off hard, resolved jet splittings via radiation and subsequent broadening of soft gluons and elastic drag. The formalism introduced in this work can be systematically improved and will be applied to other jet substructure observables in heavy-ion collisions in the future.

While the error bands provided in the plots above stem mainly from the constraining power of the experimental data at $R=0.4$ on the medium coupling $g_{\text {med }}$, we would currently like to discuss the sensitivity of our results on the various assumptions made in the setup in order to identify the main sources of uncertainty. Our main findings are summarized in Table I for moderate cone sizes $0.2 \leq R \leq 0.6$; see also Supplemental Material [74] for a full scan of parameters and their centrality dependence. First, the inclusion of the higher-twist radiative spectrum in the IOE is of mild importance for this observable, since such emissions typically occur at small angles, but it improves the description at high $p_{T}$. Furthermore, as expected, notable bias effects can be identified through the strong sensitivity to the power of the steeply falling spectrum $n$, which point to the importance of higher order terms in the large $n$ expansion that can be calculated systematically. More importantly, comparing the effect of changing the hard phase space (through $\theta_{c}$ ) and the parameters governing the behavior and recovery of soft gluons (through $\omega_{s}$ and $R_{\text {rec }}$ ), we note that an increased precision in the perturbative sector is still needed before the sensitivity to nonperturbative effects start to dominate. For instance, going beyond leading logarithmic accuracy to compute $\theta_{c}$ will be important to rigorously study the interesting marked centrality dependence of this critical angle. The importance of the recovery parameter $R_{\text {rec }}$ has been gauged between two limiting scenarios of $R_{\text {rec }}=1$ corresponding to almost complete energy recovery for large- $R$ jets and $R_{\text {rec }}=\infty$ corresponding to no energy recovery. Surprisingly, we find relatively little sensitivity to this parameter at these moderate cone sizes. Conversely, the sensitivity becomes the dominant source of uncertainty only at large- $R$, i.e., $R \approx 1$, jets.

These results emphasize the importance of analytical tools to guide more sophisticated numerical models, such as MC parton showers that, also, are better suited to incorporate the nonperturbative aspects of jet physics and quenching.
Y. M.-T. was supported by the U.S. Department of Energy, Office of Science, Office of Nuclear Physics, under Contract No. DE- SC0012704, by Laboratory Directed Research and Development (LDRD) funds from Brookhaven Science Associates and by the RHIC Physics Fellow Program of the RIKEN BNL Research Center. K. T. and D. P. are supported by a Starting Grant from Trond Mohn Foundation (BFS2018REK01) and the University of Bergen.

*mehtartani@bnl.gov

†daniel.pablos@uib.no

*konrad.tywoniuk@uib.no

[1] G. P. Salam, Eur. Phys. J. C 67, 637 (2010).

[2] A. J. Larkoski, I. Moult, and B. Nachman, Phys. Rep. 841, 1 (2020).

[3] A. M. Sirunyan et al. (CMS Collaboration), Phys. Rev. Lett. 120, 071802 (2018).

[4] M. Gyulassy and M. Plumer, Phys. Lett. B 243, 432 (1990).

[5] X.-N. Wang and M. Gyulassy, Phys. Rev. Lett. 68, 1480 (1992).

[6] K. Adcox et al. (PHENIX Collaboration), Phys. Rev. Lett. 88, 022301 (2001).

[7] C. Adler et al. (STAR Collaboration), Phys. Rev. Lett. 89, 202301 (2002).

[8] C. Adler et al. (STAR Collaboration), Phys. Rev. Lett. 90, 082302 (2003).

[9] S. Chatrchyan et al. (CMS Collaboration), Eur. Phys. J. C 72, 1945 (2012).

[10] G. Aad et al. (ATLAS Collaboration), Phys. Lett. B 719, 220 (2013).

[11] G. Aad et al. (ATLAS Collaboration), J. High Energy Phys. 09 (2015) 050.

[12] K. Aamodt et al. (ALICE Collaboration), Phys. Lett. B 696, 30 (2011).

[13] G. Aad et al. (ATLAS Collaboration), Phys. Rev. Lett. 114, 072302 (2015).

[14] J. Adam et al. (ALICE Collaboration), Phys. Lett. B 746, 1 (2015).

[15] V. Khachatryan (CMS Collaboration), Phys. Rev. C 96, 015202 (2017).

[16] B. Zakharov, JETP Lett. 63, 952 (1996).

[17] R. Baier, Y. L. Dokshitzer, A. H. Mueller, S. Peigne, and D. Schiff, Nucl. Phys. B484, 265 (1997).

[18] R. Baier, Y. L. Dokshitzer, A. H. Mueller, S. Peigne, and D. Schiff, Nucl. Phys. B483, 291 (1997).

[19] B. Zakharov, JETP Lett. 65, 615 (1997).

[20] R. Baier, Y. L. Dokshitzer, A. H. Mueller, and D. Schiff, Nucl. Phys. B531, 403 (1998).

[21] S. Caron-Huot and C. Gale, Phys. Rev. C 82, 064902 (2010).

[22] X. Feal and R. A. Vazquez, Phys. Rev. D 98, 074029 (2018).

[23] C. Andres, L. Apolinário, and F. Dominguez, J. High Energy Phys. 07 (2020) 114.

[24] I. Vitev and B.-W. Zhang, Phys. Rev. Lett. 104, 132001 (2010). 
[25] Y.-T. Chien and I. Vitev, J. High Energy Phys. 05 (2016) 023.

[26] I. Lokhtin and A. Snigirev, Eur. Phys. J. C 45, 211 (2006).

[27] K. Zapp, G. Ingelman, J. Rathsman, J. Stachel, and U. A. Wiedemann, Eur. Phys. J. C 60, 617 (2009).

[28] N. Armesto, L. Cunqueiro, and C. A. Salgado, Eur. Phys. J. C 63, 679 (2009).

[29] T. Renk, Int. J. Mod. Phys. E 20, 1594 (2011).

[30] C. Young, B. Schenke, S. Jeon, and C. Gale, Phys. Rev. C 86, 034905 (2012).

[31] J. Casalderrey-Solana, D. C. Gulhan, J. G. Milhano, D. Pablos, and K. Rajagopal, J. High Energy Phys. 10 (2014) 019; 09 (2015) 175(E).

[32] Y. He, T. Luo, X.-N. Wang, and Y. Zhu, Phys. Rev. C 91, 054908 (2015); 97, 019902(E) (2018).

[33] S. Cao, C. Park, R. A. Barbieri, S. A. Bass, D. Bazow et al. (JETSCAPE Collaboration), Phys. Rev. C 96, 024909 (2017).

[34] J. Putschke et al., arXiv:1903.07706.

[35] P. Caucal, E. Iancu, and G. Soyez, J. High Energy Phys. 10 (2019) 273.

[36] W. Ke and X.-N. Wang, J. High Energy Phys. 05 (2021) 041.

[37] Y. Mehtar-Tani, C. A. Salgado, and K. Tywoniuk, Phys. Lett. B 707, 156 (2012).

[38] Y. Mehtar-Tani and K. Tywoniuk, J. High Energy Phys. 01 (2013) 031.

[39] J. Casalderrey-Solana and E. Iancu, J. High Energy Phys. 08 (2011) 015.

[40] Y. Mehtar-Tani, C. A. Salgado, and K. Tywoniuk, J. High Energy Phys. 10 (2012) 197.

[41] J. Casalderrey-Solana, D. Pablos, and K. Tywoniuk, J. High Energy Phys. 11 (2016) 174.

[42] Y. Mehtar-Tani and K. Tywoniuk, Phys. Rev. D 98, 051501 (R) (2018).

[43] P. Caucal, E. Iancu, A. H. Mueller, and G. Soyez, Phys. Rev. Lett. 120, 232001 (2018).

[44] M. Dasgupta, F. Dreyer, G. P. Salam, and G. Soyez, J. High Energy Phys. 04 (2015) 039.

[45] M. Dasgupta, F. A. Dreyer, G. P. Salam, and G. Soyez, J. High Energy Phys. 06 (2016) 057.

[46] Z.-B. Kang, F. Ringer, and I. Vitev, J. High Energy Phys. 10 (2016) 125.

[47] L. Dai, C. Kim, and A. K. Leibovich, Phys. Rev. D 94, 114023 (2016).

[48] R. Baier, Y. L. Dokshitzer, A. H. Mueller, and D. Schiff, J. High Energy Phys. 09 (2001) 033.

[49] J.-W. Qiu, F. Ringer, N. Sato, and P. Zurita, Phys. Rev. Lett. 122, 252301 (2019).

[50] Y. He, L.-G. Pang, and X.-N. Wang, Phys. Rev. Lett. 122, 252302 (2019).

[51] C. A. Salgado and U. A. Wiedemann, Phys. Rev. D 68, 014008 (2003).

[52] A. Majumder, Phys. Rev. C 80, 031902(R) (2009).

[53] G. D. Moore and D. Teaney, Phys. Rev. C 71, 064904 (2005).

[54] J.-P. Blaizot, Y. Mehtar-Tani, and M. A. C. Torres, Phys. Rev. Lett. 114, 222002 (2015).

[55] J.-P. Blaizot, L. Fister, and Y. Mehtar-Tani, Nucl. Phys. A940, 67 (2015).
[56] E. Iancu and B. Wu, J. High Energy Phys. 10 (2015) 155.

[57] Y. Mehtar-Tani and S. Schlichting, J. High Energy Phys. 09 (2018) 144.

[58] S. Schlichting and I. Soudi, J. High Energy Phys. 07 (2021) 077.

[59] Y. Mehtar-Tani, J. High Energy Phys. 07 (2019) 057.

[60] Y. Mehtar-Tani and K. Tywoniuk, J. High Energy Phys. 06 (2020) 187.

[61] J. Barata and Y. Mehtar-Tani, J. High Energy Phys. 10 (2020) 176.

[62] M. Gyulassy, P. Levai, and I. Vitev, Phys. Rev. Lett. 85, 5535 (2000).

[63] X.-f. Guo and X.-N. Wang, Phys. Rev. Lett. 85, 3591 (2000).

[64] X. Feal, C. A. Salgado, and R. A. Vazquez, Phys. Lett. B 816, 136251 (2021).

[65] C. Andres, F. Dominguez, and M. Gonzalez Martinez, J. High Energy Phys. 03 (2021) 102.

[66] The running coupling is evaluated at leading order with five active flavors, and regularized as $\alpha_{s}(k)=$ $\min \left[1,2 \pi /\left(\beta_{0} \log k / Q_{0}\right)\right]$, with $\beta_{0}=23 / 3$ and $Q_{0}=$ $0.09 \mathrm{GeV}$. Furthermore, the Debye mass $m_{D}$ computed at LO in a thermal medium reads $m_{D}^{2}=3 g_{\text {med }}^{2} T^{2} / 2$ (for three active quark flavors).

[67] In order to simplify the numerics, such logarithmic terms are truncated at 1.

[68] P. B. Arnold and C. Dogan, Phys. Rev. D 78, 065008 (2008).

[69] A. V. Sadofyev, M. D. Sievert, and I. Vitev, arXiv: 2104.09513.

[70] S. Hauksson, S. Jeon, and C. Gale, arXiv:2109.04575.

[71] L. Antiporda, J. Bahder, H. Rahman, and M. D. Sievert, arXiv:2110.03590.

[72] J.-P. Blaizot, E. Iancu, and Y. Mehtar-Tani, Phys. Rev. Lett. 111, 052001 (2013).

[73] J. Barata, Y. Mehtar-Tani, A. Soto-Ontoso, and K. Tywoniuk, Phys. Rev. D 104, 054047 (2021).

[74] See Supplemental Material at http://link.aps.org/ supplemental/10.1103/PhysRevLett.127.252301 for a discussion of the fully differential radiative spectrum, comparison between the bare and resummed quenching weights, implementation of the medium geometry and the model uncertainties analysis, which includes [75-82].

[75] J.-P. Blaizot, F. Dominguez, E. Iancu, and Y. Mehtar-Tani, J. High Energy Phys. 01 (2013) 143.

[76] M. L. Miller, K. Reygers, S. J. Sanders, and P. Steinberg, Annu. Rev. Nucl. Part. Sci. 57, 205 (2007).

[77] W. Cassing, K. Gallmeister, and C. Greiner, Nucl. Phys. A735, 277 (2004).

[78] K. Werner, Phys. Rev. Lett. 109, 102301 (2012).

[79] C. Bierlich, G. Gustafson, L. Lönnblad, and H. Shah, J. High Energy Phys. 10 (2018) 134.

[80] P. Dorau, J.-B. Rose, D. Pablos, and H. Elfner, Phys. Rev. C 101, 035208 (2020).

[81] J. Casalderrey-Solana, D. C. Gulhan, J. G. Milhano, D. Pablos, and K. Rajagopal, J. High Energy Phys. 03 (2016) 053.

[82] R. Baier, A. Mueller, and D. Schiff, Phys. Lett. B 649, 147 (2007). 
[83] The fate of the energy and momentum deposited in the flowing plasma is currently under very active investigation. Recent studies show that the distributions of the soft hadrons coming from the wake excited by the jet passage depend on the local background flow [84,85]; others point to the importance of the interplay between the two wakes of a dijet system [86,87]. While the implications of these novel observations on our current calculation deserve a deeper study, for the purpose of the present work our ignorance on such nonperturbative effects is encapsulated in the variation of the parameter $R_{\text {rec }}$.

[84] J. Casalderrey-Solana, J. G. Milhano, D. Pablos, K. Rajagopal, and X. Yao, J. High Energy Phys. 05 (2021) 230.

[85] Y. Tachibana, C. Shen, and A. Majumder, arXiv: 2001.08321.

[86] D. Pablos, Phys. Rev. Lett. 124, 052301 (2020).

[87] L. Yan, S. Jeon, and C. Gale, Phys. Rev. C 97, 034914 (2018).

[88] U. A. Wiedemann, Nucl. Phys. B588, 303 (2000).

[89] J. Casalderrey-Solana, D. Gulhan, G. Milhano, D. Pablos, and K. Rajagopal, J. High Energy Phys. 03 (2017) 135.

[90] M. Aaboud et al. (ATLAS Collaboration), Phys. Lett. B 790, 108 (2019).

[91] In practice, we fit the shape of the initial spectra using the event generator PYTHIA [92,93], using EPS09 at LO [94] nuclear PDFs for the medium case, where the spectrum at $R=1$ was parametrized as $d \hat{\sigma}^{(k)} / d p_{T}=\sigma_{0}^{(k)}\left(p_{T, 0}^{(k)} / p_{T}\right)^{n^{(k)}\left(p_{T}\right)}$ and
$n^{(k)}\left(p_{T}\right)=\sum_{i=0}^{5} c_{i}^{(k)} \log ^{i}\left(p_{T, 0}^{(k)} / p_{T}\right)$. We then use the LO DGLAP evolution equations to obtain the spectra for $R<1$.

[92] T. Sjostrand, S. Mrenna, and P. Z. Skands, J. High Energy Phys. 05 (2006) 026.

[93] T. Sjostrand, S. Mrenna, and P. Z. Skands, Comput. Phys. Commun. 178, 852 (2008).

[94] K. Eskola, H. Paukkunen, and C. Salgado, J. High Energy Phys. 04 (2009) 065.

[95] C. Shen, Z. Qiu, H. Song, J. Bernhard, S. Bass, and U. Heinz, Comput. Phys. Commun. 199, 61 (2016).

[96] K. M. Burke, A. Buzzatti, N. Chang, C. Gale, M. Gyulassy et al. (JET Collaboration), Phys. Rev. C 90, 014909 (2014).

[97] S. Cao, Y. Chen, J. Coleman, J. Mulligan, P. M. Jacobs et al. (JETSCAPE Collaboration), Phys. Rev. C 104, 024905 (2021).

[98] A. Huss, A. Kurkela, A. Mazeliauskas, R. Paatelainen, W. van der Schee, and U. A. Wiedemann, Phys. Rev. Lett. 126, 192301 (2021).

[99] P. Caucal, E. Iancu, and G. Soyez, J. High Energy Phys. 04 (2021) 209.

[100] S. Acharya et al. (ALICE Collaboration), Phys. Rev. C 101, 034911 (2020).

[101] CMS Collaboration, Measurement of jet nuclear modification factor in $\mathrm{PbPb}$ collisions at $\sqrt{s_{N N}}=5.02 \mathrm{TeV}$ with CMS, Technical Report No. CMS-PAS-HIN-18-014 (CERN, Geneva, 2019). 